\title{
Focal low dose-rate brachytherapy for low to intermediate risk prostate cancer: preliminary experience at an Australian institution
}

\author{
Elliot Anderson', Lloyd M. L. Smyth ${ }^{2}$, Richard O'Sullivan ${ }^{3,4}$, Andrew Ryan ${ }^{5}$, Nathan Lawrentschuk ${ }^{6,7,8,9}$, \\ Jeremy Grummet ${ }^{1,10}$, Andrew W. See ${ }^{2}$ \\ ${ }^{1}$ Department of Surgery, Central Clinical School, Monash University, Melbourne, Australia; ${ }^{2}$ Icon Cancer Centre, Richmond, Australia; ${ }^{3}$ Healthcare \\ Imaging Services, Richmond, Australia; ${ }^{4}$ Department of Medicine, Monash University, Melbourne, Australia; ${ }^{5}$ TissuPath Specialist Pathology \\ Services, Mount Waverley, Australia; ${ }^{6}$ Division of Cancer Surgery, Peter MacCallum Cancer Centre, Melbourne, Australia; ${ }^{7}$ Department of Urology, \\ Royal Melbourne Hospital, Melbourne, Australia; ${ }^{8}$ Department of Surgery, University of Melbourne, Melbourne, Australia; ${ }^{9} \mathrm{EJ}$ Whitten Centre for \\ Prostate Cancer Research, Epworth Healthcare, Melbourne, Australia; ${ }^{10}$ Epworth Healthcare, Richmond, Australia \\ Contributions: (I) Conception and design: E Anderson, LML Smyth, J Grummet, AW See; (II) Administrative support: AW See, J Grummet; (III) \\ Provision of study materials or patients: J Grummet, AW See; (IV) Collection and assembly of data: E Anderson, LML Smyth; (V) Data analysis and \\ interpretation: A Ryan, R O’Sullivan; (VI) Manuscript writing: All authors; (VII) Final approval of manuscript: All authors. \\ Correspondence to: Elliot Anderson. Central Clinical School, The Alfred Centre 99 Commercial Rd, Melbourne 3004, Australia. \\ Email: elliotpeteranderson@gmail.com.
}

Background: Focal treatment for prostate cancer $(\mathrm{PCa})$ is a hybrid approach combining ablative treatment of the involved prostate gland and continued active surveillance (AS) of the unaffected gland. Low dose-rate (LDR) brachytherapy can be used as a lesion-targeted focal therapy, however, further studies are required to support its use. The aim of this study is to evaluate the dosimetry, toxicity and oncological outcomes of men receiving lesion-targeted focal LDR brachytherapy for low to intermediate risk PCa.

Methods: This is a retrospective cohort study of 26 men with unifocal, low to intermediate grade PCa diagnosed on a combination of multiparametric-magnetic resonance imaging (mp-MRI) and targeted plus template transperineal (TP) biopsy, who received focal LDR brachytherapy at a single institution. Brachytherapy involved a single monotherapy implant using iodine-125 seeds to deliver a prescribed dose of 145 Gy to the index lesion.

Results: The mean focal planning target volume (F-PTV) as a percentage of the prostate volume was $24.5 \%$. The percentage of the focal gross tumour volume (F-GTV) receiving $100 \%$ of the prescription dose was $100 \%$ for 12 patients and $\geq 98 \%$ for 18 patients. The median follow-up for toxicity and biochemical control outcomes was 23.1 [interquartile range (IQR) 19.1-31.3] and 24.2 (IQR 17.9-30.0) months, respectively. Grade 2 urinary and erectile toxicities were reported by $29.2 \%$ and $45.8 \%$ of patients, respectively, with resolution of urinary symptoms to baseline by last follow-up. There were no grade $\geq 3$ urinary or erectile toxicities or grade $\geq 2$ rectal toxicity. All 21 patients who underwent a repeat mp-MRI and TP biopsy at 12-24 months post-treatment were negative for clinically significant disease and 25 (96.2\%) patients were free from biochemical failure (FFBF).

Conclusions: Focal LDR brachytherapy is associated with a favourable toxicity profile and a high rate of control of significant PCa at 12-18 months post-treatment. We have commenced the LIBERATE prospective registry in focal LDR brachytherapy based on the highly encouraging outcomes of this initial experience.

Keywords: Brachytherapy; focal therapy; magnetic resonance imaging (MRI); prostate cancer (PCa)

Submitted Jun 06, 2021. Accepted for publication Aug 05, 2021.

doi: $10.21037 /$ tau-21-508

View this article at: https://dx.doi.org/10.21037/tau-21-508 


\section{Introduction}

Prostate cancer $(\mathrm{PCa})$ is the most common malignancy in men, contributing $25 \%$ of all new cancer cases and $12 \%$ of all cancer-related deaths in Australian males in 2019 (1). Organ-confined PCa is typically managed with radical prostatectomy $(\mathrm{RP})$ or radiation therapy which target the entire prostate gland and are associated with substantial impairment to erectile, urinary and bowel function $(2,3)$. To avoid or delay morbidity from treatment, men with low to intermediate risk PCa may be placed on an active surveillance (AS) protocol, reserving definitive treatment until disease progression has been identified by routine monitoring. The main drawbacks of AS are the potential to miss the opportunity for curative treatment and the substantial psychosocial stress associated with living with untreated $\mathrm{PCa}$ (4).

Focal therapy has emerged as a hybrid approach which involves ablative treatment of the involved prostate gland and continued AS of the unaffected gland (5). Therapies described as focal for PCa can range from treatment targeted specifically to the lesion only up to any treatment that is to less than the whole gland, such as hemi-gland ablation (6). However, as described in our series below, it is the lesion-targeted approach that takes advantage of recent advances in cancer imaging, image-guided biopsies and precision treatment delivery (7).

Scardino et al. (8), supported by the histopathologic observations of Ohori et al. (9), first proposed that targeted ablation of the "index" (or largest) PCa lesion might be sufficient for PCa control. This hypothesis is further supported by genomic analyses suggesting a monoclonal heritage for lethal metastatic disease (10), even though $\mathrm{PCa}$ is typically multifocal at presentation. Therefore, focal treatment of the index lesion, assuming that all other non-index lesions are low-grade, should be as effective as treating the whole prostate but with far less toxicity (11).

A variety of modalities, including high intensity focussed ultrasound, cryoablation, and photodynamic therapy are currently being investigated to deliver focal therapy for PCa (11). Radiotherapy in the form of low dose-rate (LDR) brachytherapy has also been adopted given its wellrecognised place as a standard option for whole-gland treatment of low to intermediate risk $\mathrm{PCa}$ (12).

Despite the increasing uptake of focal therapy for PCa across the globe, including LDR brachytherapy, robust evidence to support its efficacy and optimal utilisation is still maturing and further studies are urgently required (13). Data specifically for lesion-targeted focal
LDR brachytherapy is particularly lacking, with only six small studies (total of 115 patients) published to date (Table 1) (14-19).

This study reviews our initial experience with focal LDR brachytherapy for low to intermediate risk PCa, adding important oncological and toxicity data to the existing literature in this field and providing a preview of our subsequent prospective registry. We present the following article in accordance with the STROBE reporting checklist (available at https://dx.doi.org/10.21037/tau-21-508).

\section{Methods}

\section{Study design and patients}

This is a retrospective analysis of the electronic medical records of men who were treated with focal LDR brachytherapy between August 2015 and December 2019 at a single institution. The study was conducted in accordance with the Declaration of Helsinki (as revised in 2013). The study was approved by the Monash Health Human Research Ethics Committee (RES-20-0000-884L). The requirement for consent was waived given the retrospective nature of this study.

Twenty-six consecutive patients were included in the analysis. Patients eligible for focal LDR brachytherapy were aged 50 to 85 , with a life expectancy greater than 10 years based on comorbidities not related to PCa and with no significant obstructive urinary symptoms. Eligible patients presented with clinical stage T1c or T2a disease, a serum prostate-specific antigen (PSA) level $\leq 15 \mathrm{ng} / \mathrm{mL}$ and a lesion visible on multiparametric-magnetic resonance imaging (mp-MRI) with a prostate imaging-reporting and data system (PIRADS) score of 3-5 or a suspicious lesion on a ${ }^{68} \mathrm{Ga}$-prostate specific membrane antigen positron emission tomography $\left({ }^{68} \mathrm{Ga}\right.$-PSMA-PET) scan. In addition, patients were required to have a reproducible target plus template transperineal (TP) biopsy of the prostate gland demonstrating a histologically proven index lesion of adenocarcinoma with ISUP Grade Group $1(\geq 10 \mathrm{~mm}$ in $\geq 1$ core) or Grade Group 2 (longest core $<15 \mathrm{~mm}$ ) coincident with the radiologically visible lesion, and either no cancer, or clinically insignificant cancer (ISUP Grade Group 1 with core length $<10 \mathrm{~mm}$ ), in the remaining prostate gland.

\section{Pre-treatment staging}

MRI images were captured using a 3.0 Tesla MRI machine under PIRADS v.2 conditions. Multiple sequences 


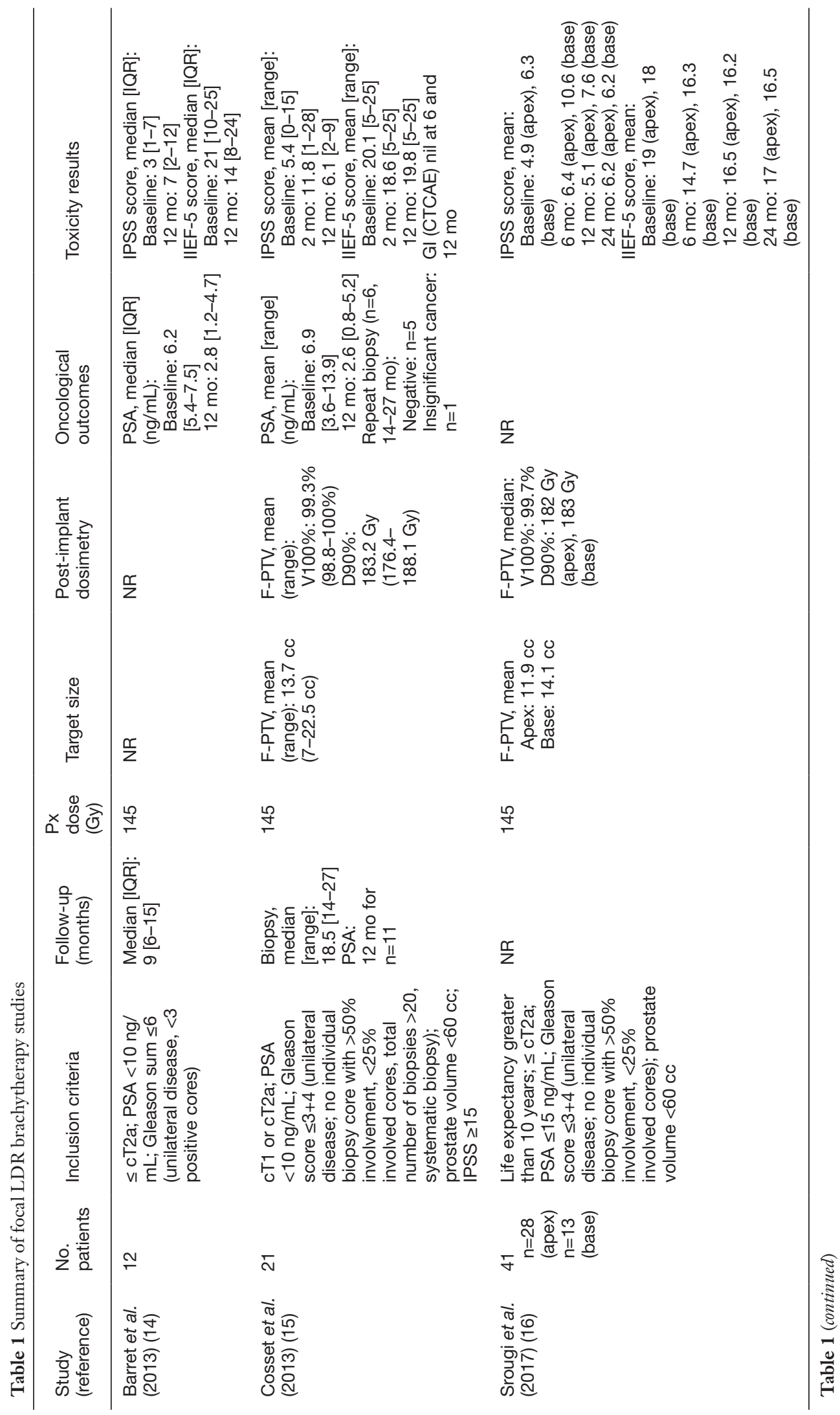




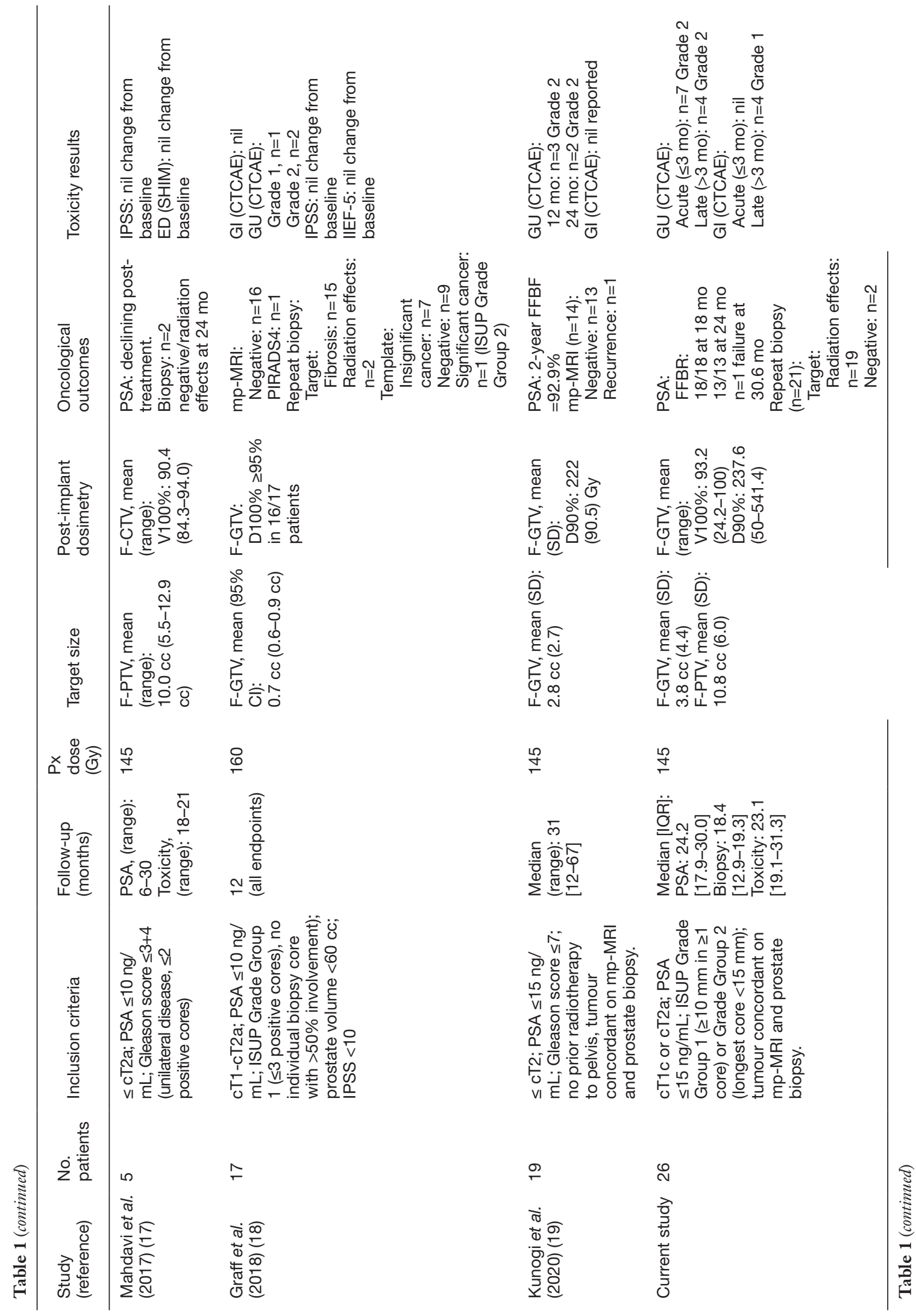




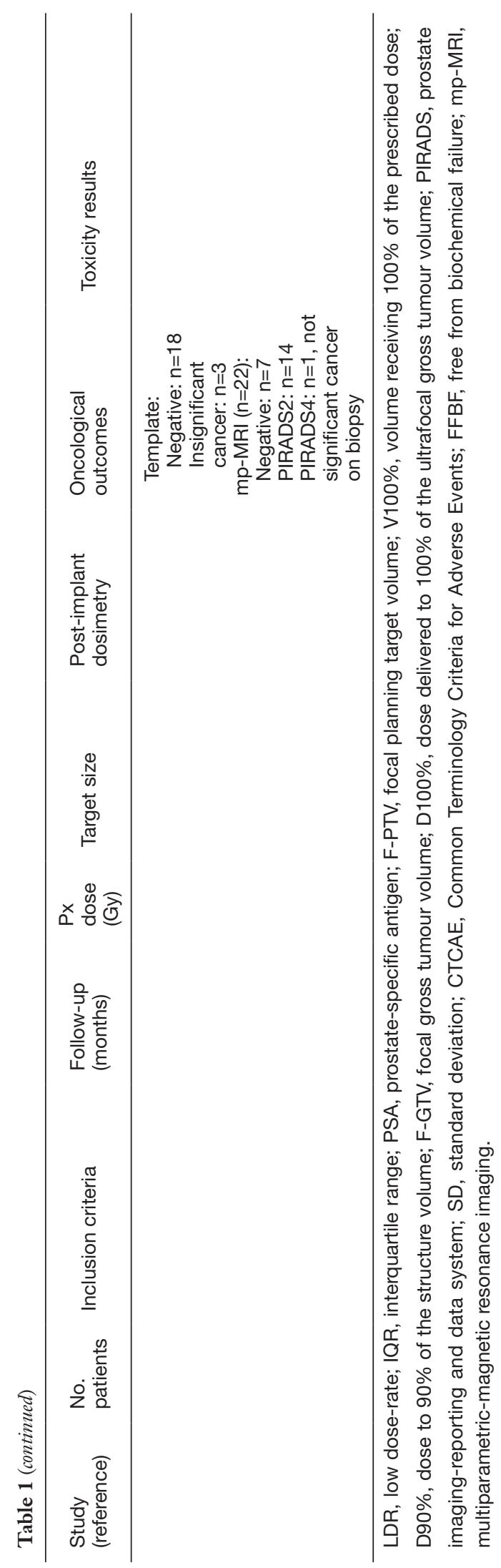

were obtained, including T2-weighted images in axial, coronal and sagittal planes, axial and sagittal diffusionweighted images including ADC map and high B value of $1,400 \mathrm{~s} / \mathrm{mm}^{2}$ and T1-weighted images of the pelvis. An axial dynamic contrast enhancement series was captured where available. All images were reviewed by an experienced radiologist who at a minimum reported on prostate size, total PIRADS score, extracapsular extension status, and size and location of all lesions.

Diagnostic TP biopsies were performed under general anaesthetic using a conventional $5 \mathrm{~mm}$ brachytherapy template grid and transrectal ultrasound (TRUS) probe. Identified MRI lesions were targeted with cognitive fusion and template cores were taken using the Ginsburg protocol (20). All biopsy samples were double-read by experienced uropathologists.

\section{Focal LDR brachytherapy}

Focal LDR brachytherapy was delivered via a standard three-phase implant technique: pre-planning seed distribution, seed implantation and analysis of the dosimetric outcomes approximately 30 days postimplantation.

All patients underwent a pre-plan volume study using TRUS 2 weeks prior to their treatment. This enabled identification of the pubic arch, urethra and rectum allowing for better seed placement and reduced toxicity. Fusion of the patient's pre-planning ultrasound and their pre-biopsy mp-MRI was performed for contouring using the fusion module within VariSeed (Varian Medical Systems, Palo Alto, CA, USA) by a senior radiation oncologist and verified by a senior radiation therapist or radiation oncology medical physicist. The focal gross tumour volume (F-GTV) was the radiological extent of the index lesion, defined by a Boolean addition of the areas of abnormality observed on the different mp-MRI sequences captured and the ${ }^{68} \mathrm{Ga}$-PSMAPET scan, if performed. The focal planning target volume (F-PTV) was a $7 \mathrm{~mm}$ isotropic expansion of the F-GTV to account for systematic uncertainties inherent within imaging modalities and post-acquisition image manipulation including fusion. For posteriorly located lesions adjacent to the rectum, the posterior GTV-PTV expansion was $0 \mathrm{~mm}$. Eighteen men, all of whom had posterior index lesions, also received a SpaceOAR ${ }^{\circledR}$ (Boston Scientific, Malborough, MA, USA) gel implant between the anterior rectal wall and whole prostate.

Focal LDR brachytherapy consisted of a single 
monotherapy implant delivering a prescribed dose of 145 Gy to the F-PTV. Treatment was performed by an experienced brachytherapist. Iodine-125 Amersham brachytherapy seeds (model 6711) in a range of activities from $(0.311-0.500 \mathrm{mCi})$ were utilised. Implantation was performed under general anaesthetic with patients in extended lithotomy position. Seeds were placed as per the pre-plan set-up under ultrasound guidance. A minimum distance of $3 \mathrm{~mm}$ was maintained between seeds and the urethra, which was demarcated with an aerated gel. Intraoperative real-time dosimetric analysis was conducted within the VariSeed suite. Additional 'zulu' (free) seeds, were inserted if any clinically meaningful deviation from the intended plan was suspected. A non-contrast pelvic CT scan, co-registered with a same day mp-MRI scan, was obtained 30 days post-implant in order to assess dosimetric outcomes. Follow-up occurred 4-6 weeks after seed implant and then at three- to six-monthly intervals thereafter. Reviews included a clinical exam, PSA test and toxicity assessment.

\section{Outcome measures}

To assess post-implantation dosimetry, the volume receiving $100 \%$ of the prescribed dose (V100\%), volume receiving $150 \%$ of the prescribed dose (V150\%) and dose to $90 \%$ of the structure volume (D90\%) for the F-GTV, V100\% for the whole prostate, volume receiving $200 \%$ of the prescribed dose (V200\%) for the urethra and V100\% for the rectum were collected.

Baseline and post-treatment symptoms described in clinician notes were grouped under urinary, rectal and erectile domains and toxicity was assessed by retrospectively grading these according to the system used in the Common Terminology Criteria for Adverse Events (CTCAE, version 5.0):

(I) Grade 1-mild; asymptomatic or mild symptoms; intervention not indicated.

(II) Grade 2-moderate; minimal, local or non-invasive indication indicated.

(III) Grade 3-severe or medically significant but not life threatening; hospitalisation indicated.

Oncological outcomes were assessed via serial PSA results and the findings of repeat mp-MRI and TP biopsy which were performed 12-18 months post-treatment. The target region for the repeat TP biopsy was based on the lesion visible on the pre-treatment mp-MRI.

\section{Statistical analysis}

Analyses were performed in GraphPad Prism (v8.4.1). Numerical variables are presented as a median [interquartile range (IQR)] or mean (range), as specified. Frequencies are reported as a number and percentage of the assessable patients for a given outcome.

\section{Results}

\section{Patient characteristics}

Baseline patient characteristics are described in Table 2. All patients had unifocal disease on mp-MRI, low to intermediate grade tumours (ISUP Grade Group 1 or 2) and a risk of nodal disease lower than $15 \%$ based on Kattan nomograms (21). No patients received androgen deprivation therapy prior to, or at the time of, treatment. One patient was ineligible for mp-MRI due to the presence of bilateral hip prostheses but had a targetable unifocal lesion on a ${ }^{68}$ Ga-PSMA-PET scan.

\section{Dosimetry}

Intra- and post-operative dosimetry outcomes are summarised in Table 3. The mean (range) operating time for the seed insertion procedure was $36 \mathrm{~min}$ (23-47 $\mathrm{min}$ ). The majority of men 24 (92.3\%) were discharged on the day of treatment, with the remainder staying overnight for social reasons. All men passed their trial of void prior to discharge.

The mean (range) post-implantation V100\% (Figure $1 A$ ) and D90\% (Figure $1 B$ ) for the F-GTV were $92.3 \%(24.2-100 \%)$ and 237.6 Gy (50.0-541.4 Gy), respectively. Twelve patients had a F-GTV V100\% $=100 \%$ and 18 patients had V100\% $\geq 98 \%$. The first three consecutive patients had a F-GTV V100\% $<85 \%$, prompting a change in planning technique from traditional seed placement to end-to-end seed clustering.

Twenty men (76.9\%) had a rectal V100\% of zero, with the remaining six men having rectal V100\% $<1 \mathrm{cc}(12)$. The average (range) maximum urethral dose was 164.6 Gy (66.8-259.6 Gy) and 23 men (88.5\%) had an unrecordable V200\%. The mean (range) PTV size as a percentage of the prostate volume (PTV/prostate) was $24.5 \%(6.9-52.5 \%)$ and the prostate $\mathrm{V} 100 \%$ was $31.7 \%$ $(9.2-62.2 \%)$. 
Table 2 Patient characteristics

\begin{tabular}{|c|c|}
\hline Characteristic & $\mathrm{N}(\%)$ \\
\hline Age: mean (SD) & $71(5.6)$ \\
\hline \multicolumn{2}{|l|}{ Clinical stage } \\
\hline T1c & $17(65.4)$ \\
\hline $\mathrm{T} 2 \mathrm{a}$ & $5(19.2)$ \\
\hline Missing & $4(15.4)$ \\
\hline Pre-biopsy PSA (ng/mL): mean (SD) & $7.3(3.1)$ \\
\hline \multicolumn{2}{|l|}{ TP biopsy: median [IQR] } \\
\hline Total no. cores taken & $28[24-31]$ \\
\hline Target no. cores taken & $7[6-8]$ \\
\hline No. positive target cores & $4[3-6]$ \\
\hline Template no. cores taken & $18[18-24]$ \\
\hline No. positive template cores & $2[0-3]$ \\
\hline Longest length cancer (mm) & $7.5[5-11]$ \\
\hline \multicolumn{2}{|l|}{ ISUP grade-group } \\
\hline 1 (Gleason score 3+3) & $1(3.8)$ \\
\hline 2 (Gleason score $3+4$ ) & $25(96.2)$ \\
\hline \multicolumn{2}{|l|}{ PIRADS score } \\
\hline 3 & $1(3.8)$ \\
\hline 4 & $19(73.1)$ \\
\hline 5 & $5(19.2)$ \\
\hline Missing & $1(3.8)$ \\
\hline \multicolumn{2}{|l|}{ Lesion location } \\
\hline Base & 7 (26.9) \\
\hline Middle & $9(34.6)$ \\
\hline Apex & $9(34.6)$ \\
\hline Base to apex & $1(3.8)$ \\
\hline
\end{tabular}

IQR, interquartile range; PIRADS, prostate imaging-reporting and data system; PSA, prostate-specific antigen; SD, standard deviation; TP, transperineal.

\section{Toxicity}

The median time from treatment to last toxicity assessment was 19.0 (IQR 12.4-30.5) months. Two patients were reviewed by clinicians outside of our institution and were lost to toxicity follow-up.

One patient developed a urinary tract infection one week post-implant that was managed with oral antibiotics and one patient went into urinary retention one week following implant, requiring temporary catheterisation. There were no acute readmissions following implantation. The frequency and severity of urinary symptoms peaked within 3 months of treatment, with $9(37.5 \%)$ and 7 (29.2\%) presenting with Grade 1 and 2 urinary symptoms, respectively, which resolved predominantly to baseline levels by the time of last follow-up (Figure $2 \mathrm{~A}$ ). Six of 13 patients with a F-PTV/prostate proportion greater than $20 \%$ had a Grade 2 urinary toxicity following treatment, compared to 1 of 11 patients where the F-PTV/prostate proportion was less than $20 \%$.

Eleven $(45.8 \%)$ men reported a reduction in erectile function at any point after treatment compared to baseline, with $8(33.3 \%)$ men continuing to have worse erectile function at the time of last follow-up (Figure $2 B$ ). No Grade 3 erectile dysfunction, refractory to pharmacological intervention, was reported.

Rectal toxicity was minimal (Figure 2C) with only four (16.7\%) patients having minor (Grade 1) rectal symptoms post-treatment. One patient had Grade 1 rectal toxicity at the time of last follow up.

\section{Oncological outcomes}

At the time of analysis, 12- to 18-month oncological outcomes were available for 21 patients via mp-MRI and TP biopsy $(n=21)$ or mp-MRI only $(n=1)$. The median time to repeat TP biopsy following treatment was 18.4 months (IQR 12.9-19.3). No patients had clinically significant PCa, defined as ISUP Grade Group 2 or above. Histology results for the targeted index lesion/ treatment area showed 7 men negative for malignancy with radiation effect present, 12 men with adenocarcinoma showing radiation treatment effect with no Gleason score assigned and 2 patients negative for malignancy with no neoplastic changes visible. Eighteen patients had no cancer detected in the remainder of the nontreated prostate and 3 patients had clinically insignificant disease (ISUP Grade-Group 1 with core length $<10 \mathrm{~mm}$ ). No lesion visible on repeat mp-MRI had a PIRADS score $\geq 3$. Eight patients returned a negative result while 10 patients had a lesion with a PIRADS score equal to 2 .

The median PSA follow-up time for the cohort was 24.2 (IQR 17.9-30.0) months (Figure 3A). All patients had 
Table 3 Intra-operative and post-operative dosimetry outcomes

\begin{tabular}{|c|c|}
\hline Variable & Value \\
\hline \multicolumn{2}{|l|}{ Intra-operative } \\
\hline Number of needles: median [IQR] & $13[11-15]$ \\
\hline Number of seeds: median [IQR] & 39 [34-47] \\
\hline Total implanted activity (mCi) & $16.7[5.2]$ \\
\hline \multicolumn{2}{|l|}{ Geometry, mean (range) } \\
\hline Prostate volume (cc) & $47.0(19.3)$ \\
\hline F-GTV (cc) & $3.8(4.4)$ \\
\hline F-PTV (cc) & $10.8(6.0)$ \\
\hline F-PTV (\% of prostate volume) & $24.5(11.0)$ \\
\hline \multicolumn{2}{|l|}{ F-GTV, mean (range) } \\
\hline V100\% (\%) & $93.2(24.2-100)$ \\
\hline V150\% (\%) & $82.9(9.8-100)$ \\
\hline D90\% (Gy) & $237.6(50.0-541.4)$ \\
\hline \multicolumn{2}{|l|}{ Prostate, mean (range) } \\
\hline V100\% (\%) & $31.7(9.2-62.2)$ \\
\hline \multicolumn{2}{|l|}{ Urethra, mean (range) } \\
\hline $\operatorname{Max}(\mathrm{Gy})$ & $164.6(66.8-259.6)$ \\
\hline V200\% (cc) & $0.0(0.0-0.01)$ \\
\hline \multicolumn{2}{|l|}{ Rectum, mean (range) } \\
\hline $\operatorname{Max}(\mathrm{Gy})$ & $95.8(18.4-278.1)$ \\
\hline V100\% (cc) & $0.05(0.00-0.84)$ \\
\hline
\end{tabular}

D90\%, dose to $90 \%$ of the structure volume; F-GTV, focal gross tumour volume; F-PTV, focal planning target volume; IQR, interquartile range; SD, standard deviation; V100\%, volume receiving $100 \%$ of the prescribed dose; V150\%, volume receiving $150 \%$ of the prescribed dose; V200\%, volume receiving $200 \%$ of the prescribed dose.

a reduction in PSA following focal LDR brachytherapy, with a mean decrease in PSA from baseline at last follow-up of $72.1 \%$ (range, 21.9-95.1\%) (Figure 3B). Of the 18 patients who had reached 18 months followup, all were free from biochemical failure (FFBF) [PSA $>2$ $\mathrm{ng} / \mathrm{mL}$ above post-radiotherapy nadir (22)] (Figure 3C). At 24 months, 13 out of 13 patients were FFBF. One patient, who had a F-GTV V100\% of $97.0 \%$ and D90\% of $163.6 \mathrm{~Gy}$, and whose 12-month post-treatment biopsy had been negative, developed a rising PSA at 30 months and proceeded to an uncomplicated robotic-assisted RP. The final histopathology demonstrated an in-field recurrence of PCa (ISUP Grade Group 3) that was staged as T2N0M0 disease with clear margins.

\section{Discussion}

Advancements in modern imaging have facilitated a widespread move towards tissue-preserving strategies for cancer management, of which focal brachytherapy is an example for PCa. There are five prospective studies currently in recruitment across Europe, North America and Australia-including a clinical registry (Australian and New Zealand Trials Registry, CTRN12619001669189, LIBERATE) at our institution-investigating focal brachytherapy for selected PCa patients. This reflects an urgent need for further data to evaluate whether these techniques should be implemented more widely. While prospectively collected data continue to mature, the findings of this study affirm that lesion-targeted focal LDR brachytherapy is technically feasible, albeit with a learning curve, has a favourable toxicity profile compared to whole-gland treatments, and controls clinically significant cancer at 18-24 months following treatment.

Formal post-implant dosimetric evaluation criteria for focal LDR brachytherapy does not yet exist. Criteria for whole gland brachytherapy, such as the British Columbia Cancer Agency criteria (23), do not strictly require complete coverage of the prostate by the prescription dose, with a V100\% $>85 \%$ considered 'good' and $\geq 90 \%$ considered excellent. In contrast, in the focal setting, it is likely that near complete coverage of the focal GTV with the prescription dose will be critical. In a previous prospective trial of focal LDR brachytherapy, the criterion for a successful implant was a post-implant D $100 \% \geq 95 \%$ for the F-GTV (18). This objective was met in 16 of 17 patients, however, the mean focal GTV size was only $0.7 \mathrm{cc}$, compared to $3.8 \mathrm{cc}$ in our study. A planning objective of $\mathrm{V} 100 \% \geq 98 \%$ to the focal volume has also been used previously $(17,19)$. The post-implant dosimetry and size of the focal target volume reported in our study is comparable to that reported by Cosset et al. (15), Mahdavi et al. (17) and Kunogi et al. (19) (Table 1).

The proportion of the prostate irradiated decreases progressively from whole-gland treatment to hemi-gland and lesion-targeted focal treatment of $\mathrm{PCa}$, and with this, the rate of toxicity is also expected to decrease. On average, the PTV was one quarter of the prostate volume in our study. Rates of Grade 2 or higher acute and late urinary toxicity 

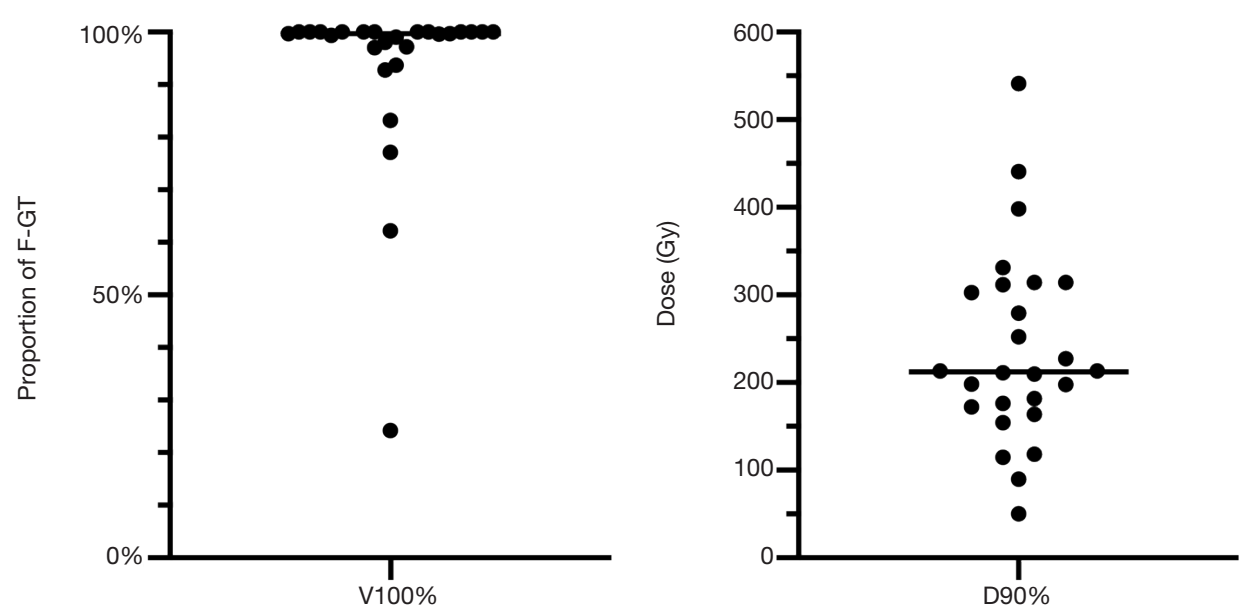

Figure 1 Post-implantation target dosimetry. The volume of the F-GTV receiving $100 \%$ of the prescription dose (A) and the dose to $90 \%$ of the F-GTV (B). V100\%, volume receiving 100\% of the prescribed dose; D90\%, dose to $90 \%$ of the structure volume; F-GTV, focal gross tumour volume.

following whole-prostate LDR brachytherapy are reported to be up to $45 \%$ and $30 \%$, respectively, including Grade 3 or higher urinary toxicity in $5-10 \%$ of patients $(24,25)$. Using a grading system aligned with the CTCAE, 29\% and 17\% of men in our study had Grade 2 acute and late urinary toxicity, respectively, with no Grade 3 toxicities reported. Other studies of focal LDR brachytherapy report the majority of urinary toxicity within the initial 6 months following treatment, mostly resolving to baseline by 12 months $(15,16)$. Our results support this trend, with the initial worsening of urinary symptoms likely to reflect an inflammatory response from seed insertion.

Predictors of toxicity following whole-gland LDR brachytherapy include the number of needles used during insertion and the prostate $\mathrm{V} 150 \%$ (24), and for focal treatment, lesions located at the base of the prostate (16). While our study was not powered to detect predictors of toxicity, there did not appear to be a relationship between needle number or lesion location and urinary toxicity. However, a PTV/prostate proportion greater than 20\% was associated with more Grade 2 urinary toxicity. In a prospective study of 17 patients treated with focal LDR brachytherapy, Graff et al. (18) report only one CTCAEdefined Grade 2 acute urinary toxicity and no late Grade 2 toxicity, which is likely explained by the substantially smaller average F-GTV size $\left(0.7\right.$ versus $3.8 \mathrm{~cm}^{3}$ in the present study) and a smaller proportion of the prostate being irradiated with the prescription dose. Taking these observations together, the F-PTV (or F-GTV) size as a proportion of the total prostate volume might be an important metric predictive of toxicity in the focal setting.

Similar to this study, the rectal dose (V100\%) and subsequent toxicity associated with focal LDR brachytherapy has been universally reported as low to negligible $(15,17,18)$. In comparison, rates of Grade 2 gastrointestinal toxicity have been reported to range from $1-19 \%$ following whole-gland LDR brachytherapy, with severe (Grade $\geq 3$ ) injuries including fistula reported in $1-2 \%$ of patients (26-28). The insertion of a rectal spacer between the prostate and anterior rectal wall, which was performed for the 18 men with posterior lesions in this study, is likely to further decrease the likelihood of rectal symptoms following focal treatment.

The rate of erectile dysfunction requiring pharmacological or mechanical intervention following whole-gland LDR brachytherapy is reported to be at least $50 \%$ (29). Initial data for focal brachytherapy suggest that erectile function returns to baseline levels for a substantial proportion of men after an initial decline in erectile function following treatment, however there is significant heterogeneity in the outcome measures used $(15,18)$. In comparison, we observed an increase in the rate of erectile dysfunction requiring pharmacological intervention at the time of last follow up compared to baseline. 
A
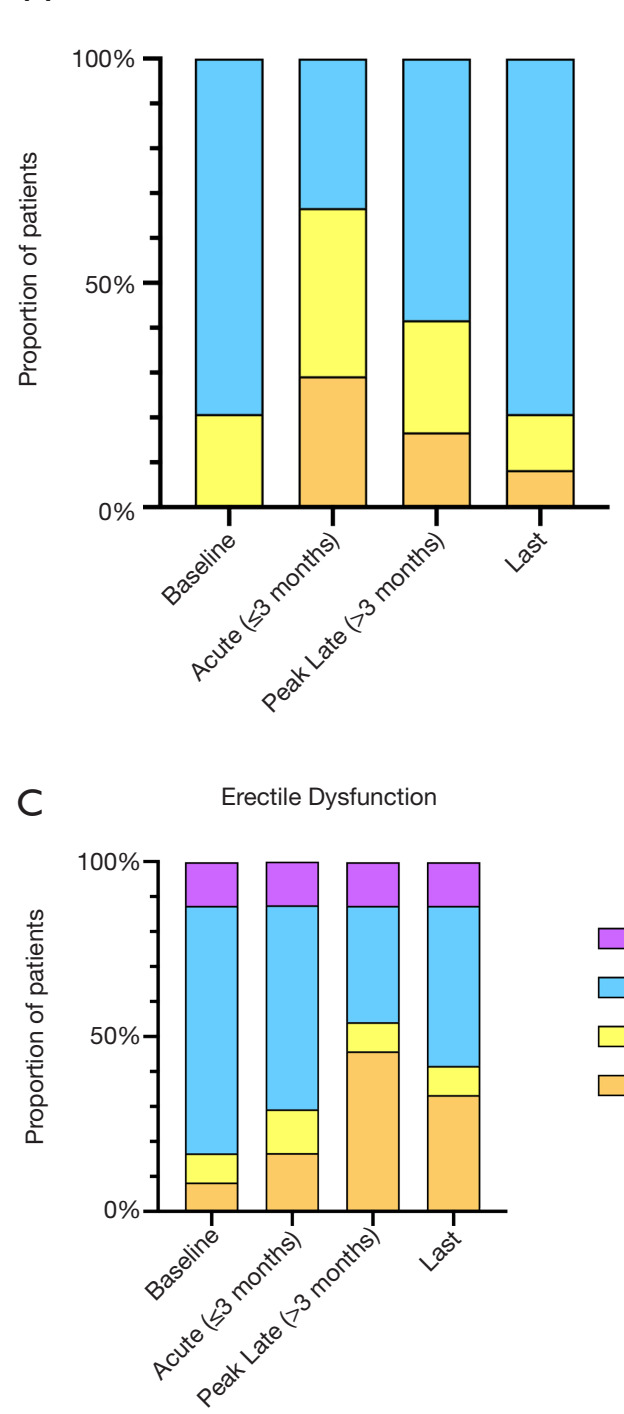

B

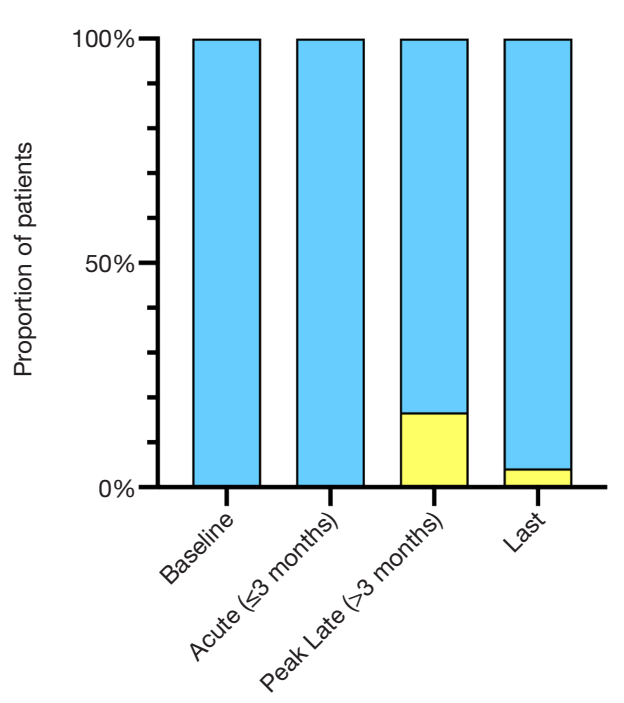

Not Sexually Active

\section{None}

Grade 1

Grade 2

Figure 2 Summary of post-treatment toxicity over time. Rates of urinary (A) toxicity peaked acutely following treatment, before resolving mostly to baseline levels by the time of last follow-up. Rectal toxicity (B) was minimal at all time points, with no Grade 2 or higher toxicities reported. Rates of erectile dysfunction (C) peaked greater than 3 months post-treatment, with a resolution of symptoms in a minority of patients by the time of last follow-up.

However, our study could not distinguish between men receiving prophylactic intervention to maintain erectile function versus those being actively treated for a decline in function, making the true rate of erectile dysfunction likely to be lower than reported. The LIBERATE registry will prospectively collect these data as well as changes in international index of erectile function (IIEF) scores over time.

The oncological outcomes in this study are promising, however, longer term follow-up is required to assess the true efficacy of lesion-targeted focal treatment. A proportion of patients will experience recurrence despite initial disease control, as did one patient in our cohort who was negative for clinically significant disease at 12 months post-treatment.

Consensus guidelines from an international multidisciplinary group recently stated that the primary objective of focal therapy clinical trials for PCa should be 

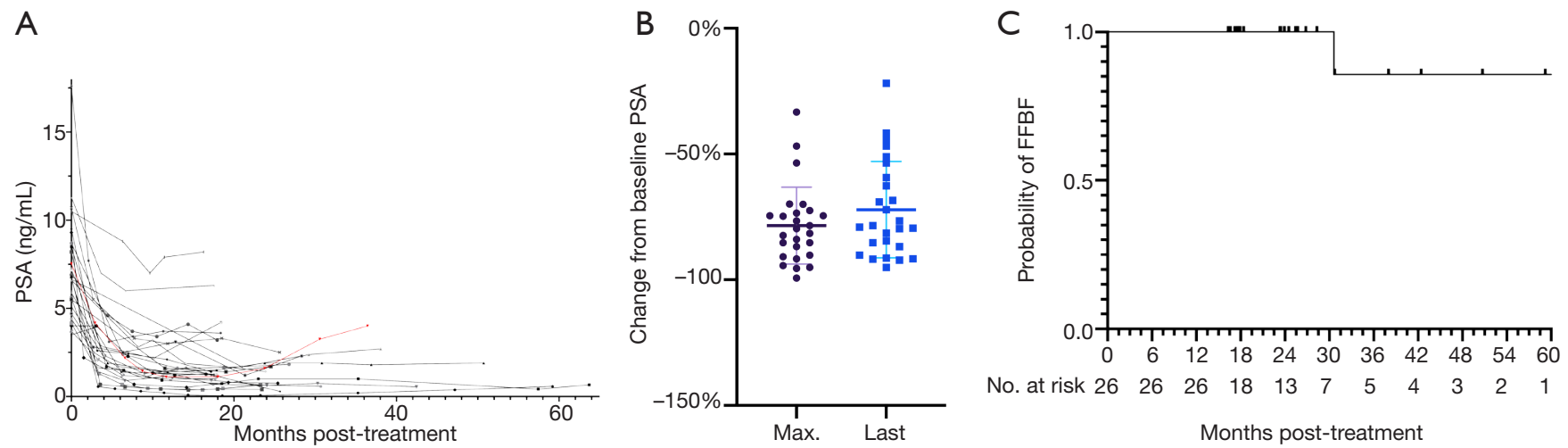

Figure 3 PSA outcomes following focal LDR brachytherapy. (A) PSA time-course for individual patients following treatment. (B) Maximum and last change in PSA from baseline. (C) Kaplan-Meier curve showing the probability of FFBF following treatment. PSA, prostate-specific antigen; LDR, low dose-rate; FFBF, free from biochemical failure.

to demonstrate the focal ablation of clinically significant disease with negative biopsies at 12 months after treatment (30). However, it is important to acknowledge that radiotherapy, histologic changes are not usually seen within 12 months of radiotherapy and complete histologic elimination of the tumour can take up to 3 years (31). Furthermore, the interpretation of prostate histology following irradiation can be difficult due to radiationinduced cytoplasmic changes in benign tissue (32). Repeat biopsies were performed at a median of 18.4 months (IQR 12.9-19.3) post-treatment in the majority of the patients in this study, in alignment with AS guidelines for PCa (33). Consistent with our study, previous studies of focal LDR brachytherapy report repeat TP biopsy results at 12 months [Graff $e t$ al. (18), $\mathrm{n}=17$ patients with all being negative], up to $18-24$ months [Cosset et al. (15), $\mathrm{n}=6$ patients with $\mathrm{n}=5$ being negative]. Madhavi et al. (17) report 24-month repeat TP biopsy results for two patients, finding no clinically significant cancer and demonstrating radiation effects in the respective focal target regions.

For patients treated with whole-gland external beam radiotherapy, patients with adenocarcinoma showing severe treatment effects at 2 to 3 years post-treatment have longterm disease-free survival equivalent to patients with a negative biopsy $(34,35)$. Further data on the relationship between histological and clinical outcomes following brachytherapy, and in particular, focal brachytherapy, are still required. The prospective LIBERATE registry, currently underway, will assess 18-month local control, based on repeat biopsy and mp-MRI, alongside 5-year biochemical progression free survival. The applicability of standard definitions of biochemical failure following whole- gland radiotherapy (22) in the focal setting may not be valid and should also be investigated.

A potential disadvantage of focal therapy is that it may increase the toxicity and rate of complications associated with future salvage therapy, if it is required (13). There is only a weak evidence to date to suggest that the rate of complications, as well as functional and oncological outcomes, are acceptable post-salvage following primary focal therapy (36). A better understanding of postsalvage treatment toxicity and oncological outcomes is a prerequisite for more widespread clinical use of focal LDR brachytherapy.

This study has several limitations. It is retrospective in nature and relatively small, lacking the power to formally interrogate predictors of toxicity following treatment at specific timepoints. Also, rates of toxicities were reported broadly under urinary and rectal domains, as it was not possible to identify specific toxicities in the medical records of all patients. Finally, many patients had a relatively short follow-up time, limiting conclusions about long-term toxicity and oncological outcomes.

\section{Conclusions}

This retrospective study contributes important data to the growing field of focal brachytherapy for PCa, which currently requires substantially more evidence to support widespread clinical implementation. We have demonstrated that focal LDR brachytherapy is safe and feasible, with encouraging preliminary oncological and functional outcomes. Prospective studies, such as the LIBERATE clinical registry at our institution, will answer crucial 
questions about the efficacy and utility of focal LDR brachytherapy, including quality of life outcomes measured by validated instruments, the impact on salvage therapy, and the correlation between repeat-biopsy and long-term biochemical control outcomes.

\section{Acknowledgments}

Part of this manuscript was presented at the Canadian Urology Association Annual Meeting 2021.

Funding: None.

\section{Footnote}

Reporting Checklist: The authors have completed the STROBE reporting checklist. Available at https://dx.doi. org/10.21037/tau-21-508

Data Sharing Statement: Available at https://dx.doi. org/10.21037/tau-21-508

Peer Review File: Available at https://dx.doi.org/10.21037/ tau-21-508

Conflict of Interest: All authors have completed the ICMJE uniform disclosure form (available at https://dx.doi. org/10.21037/tau-21-508). The authors have no conflicts of interest to declare.

Ethical Statement: The authors are accountable for all aspects of the work in ensuring that questions related to the accuracy or integrity of any part of the work are appropriately investigated and resolved. The study was conducted in accordance with the Declaration of Helsinki (as revised in 2013). This retrospective study was approved by the Monash Health Human Research Ethics Committee (RES-20-0000-884L). The requirement for consent was waived by the Monash Health Human Research Ethics Committee given the retrospective nature of this study.

Open Access Statement: This is an Open Access article distributed in accordance with the Creative Commons Attribution-NonCommercial-NoDerivs 4.0 International License (CC BY-NC-ND 4.0), which permits the noncommercial replication and distribution of the article with the strict proviso that no changes or edits are made and the original work is properly cited (including links to both the formal publication through the relevant DOI and the license).
See: https://creativecommons.org/licenses/by-nc-nd/4.0/.

\section{References}

1. Australian Institute of Health and Welfare 2019. Cancer in Australia 2019. Canberra: AIHW, 2019.

2. Resnick MJ, Koyama T, Fan KH, et al. Long-term functional outcomes after treatment for localized prostate cancer. N Engl J Med 2013;368:436-45.

3. Barocas DA, Alvarez J, Resnick MJ, et al. Association Between Radiation Therapy, Surgery, or Observation for Localized Prostate Cancer and Patient-Reported Outcomes After 3 Years. JAMA 2017;317:1126-40.

4. Davison BJ, Goldenberg SL. Patient acceptance of active surveillance as a treatment option for low-risk prostate cancer. BJU Int 2011;108:1787-93.

5. Lindner U, Trachtenberg J, Lawrentschuk N. Focal therapy in prostate cancer: modalities, findings and future considerations. Nat Rev Urol 2010;7:562-71.

6. Langley S, Ahmed HU, Al-Qaisieh B, et al. Report of a consensus meeting on focal low dose rate brachytherapy for prostate cancer. BJU Int 2012;109 Suppl 1:7-16.

7. Kasivisvanathan V, Rannikko AS, Borghi M, et al. MRITargeted or Standard Biopsy for Prostate-Cancer Diagnosis. N Engl J Med 2018;378:1767-77.

8. Scardino PT. Focal therapy for prostate cancer. Nat Rev Urol 2009;6:175.

9. Ohori M, Eastham JA, Koh H, et al. 1574: Is focal therapy reasonable in patients with early stage prostate cancer (CAP)-an analysis of radical prostatectomy (RP) specimens. J Urol 2006;175:507.

10. Liu W, Laitinen S, Khan S, et al. Copy number analysis indicates monoclonal origin of lethal metastatic prostate cancer. Nat Med 2009;15:559-65.

11. Perera M, Krishnananthan N, Lindner U, et al. An update on focal therapy for prostate cancer. Nat Rev Urol 2016;13:641-53.

12. Davis BJ, Horwitz EM, Lee WR, et al. American Brachytherapy Society consensus guidelines for transrectal ultrasound-guided permanent prostate brachytherapy. Brachytherapy 2012;11:6-19.

13. van der Poel HG, van den Bergh RCN, Briers E, et al. Focal Therapy in Primary Localised Prostate Cancer: The European Association of Urology Position in 2018. Eur Urol 2018;74:84-91.

14. Barret E, Ahallal Y, Sanchez-Salas R, et al. Morbidity of focal therapy in the treatment of localized prostate cancer. Eur Urol 2013;63:618-22. 
15. Cosset JM, Cathelineau X, Wakil G, et al. Focal brachytherapy for selected low-risk prostate cancers: a pilot study. Brachytherapy 2013;12:331-7.

16. Srougi V, Barret E, Nunes-Silva I, et al. Focal brachytherapy for localized prostate cancer: Urinary toxicity depends on tumor location. Brachytherapy 2017;16:988-92.

17. Mahdavi SS, Spadinger IT, Salcudean SE, et al. Focal application of low-dose-rate brachytherapy for prostate cancer: a pilot study. J Contemp Brachytherapy 2017;9:197-208.

18. Graff P, Portalez D, Lusque A, et al. IDEAL 2a Phase II Study of Ultrafocal Brachytherapy for Low- and Intermediate-risk Prostate Cancer. Int J Radiat Oncol Biol Phys 2018;102:903-11.

19. Kunogi H, Wakumoto Y, Kawamoto T, et al. Focal low-dose-rate prostate brachytherapy for lowand intermediate-risk prostate cancer. J Contemp Brachytherapy 2020;12:554-61.

20. Kuru TH, Wadhwa K, Chang RT, et al. Definitions of terms, processes and a minimum dataset for transperineal prostate biopsies: a standardization approach of the Ginsburg Study Group for Enhanced Prostate Diagnostics. BJU Int 2013;112:568-77.

21. Kattan MW, Eastham JA, Stapleton AM, et al. A preoperative nomogram for disease recurrence following radical prostatectomy for prostate cancer. J Natl Cancer Inst 1998;90:766-71.

22. Roach M 3rd, Hanks G, Thames H Jr, et al. Defining biochemical failure following radiotherapy with or without hormonal therapy in men with clinically localized prostate cancer: recommendations of the RTOG-ASTRO Phoenix Consensus Conference. Int J Radiat Oncol Biol Phys 2006;65:965-74.

23. Keyes M, Morris WJ, Spadinger I, et al. Radiation oncology and medical physicists quality assurance in British Columbia Cancer Agency Provincial Prostate Brachytherapy Program. Brachytherapy 2013;12:343-55.

24. Keyes M, Miller S, Moravan V, et al. Predictive factors for acute and late urinary toxicity after permanent prostate brachytherapy: long-term outcome in 712 consecutive patients. Int J Radiat Oncol Biol Phys 2009;73:1023-32.

25. Vuolukka K, Auvinen P, Palmgren JE, et al. Longterm efficacy and urological toxicity of low-dose-rate brachytherapy (LDR-BT) as monotherapy in localized prostate cancer. Brachytherapy 2019;18:583-8.

26. Stone NN, Stock RG. Complications following permanent prostate brachytherapy. Eur Urol 2002;41:427-33.

27. Cosset JM, Flam T, Belin L, et al. Long-term results of permanent implant prostate cancer brachytherapy: A single-institution study of 675 patients treated between 1999 and 2003. Cancer Radiother 2016;20:261-7.

28. Keyes M, Spadinger I, Liu M, et al. Rectal toxicity and rectal dosimetry in low-dose-rate (125)I permanent prostate implants: a long-term study in 1006 patients. Brachytherapy 2012;11:199-208.

29. Taira AV, Merrick GS, Galbreath RW, et al. Erectile function durability following permanent prostate brachytherapy. Int J Radiat Oncol Biol Phys 2009;75:639-48.

30. van den Bos W, Muller BG, Ahmed H, et al. Focal therapy in prostate cancer: international multidisciplinary consensus on trial design. Eur Urol 2014;65:1078-83.

31. Crook JM, Bahadur YA, Robertson SJ, et al. Evaluation of radiation effect, tumor differentiation, and prostate specific antigen staining in sequential prostate biopsies after external beam radiotherapy for patients with prostate carcinoma. Cancer 1997;79:81-9.

32. Cheng L, Cheville JC, Bostwick DG. Diagnosis of prostate cancer in needle biopsies after radiation therapy. Am J Surg Pathol 1999;23:1173-83.

33. Morash C, Tey R, Agbassi C, et al. Active surveillance for the management of localized prostate cancer: Guideline recommendations. Can Urol Assoc J 2015;9:171-8.

34. Zelefsky MJ, Goldman DA, Reuter V, et al. Long-Term Implications of a Positive Posttreatment Biopsy in Patients Treated with External Beam Radiotherapy for Clinically Localized Prostate Cancer. J Urol 2019;201:1127-33.

35. Crook JM, Malone S, Perry G, et al. Twenty-four-month postradiation prostate biopsies are strongly predictive of 7-year disease-free survival: results from a Canadian randomized trial. Cancer 2009;115:673-9.

36. Marra G, Gontero P, Walz JC, et al. Complications, oncological and functional outcomes of salvage treatment options following focal therapy for localized prostate cancer: a systematic review and a comprehensive narrative review. World J Urol 2019;37:1517-34.

Cite this article as: Anderson E, Smyth LML, O'Sullivan R, Ryan A, Lawrentschuk N, Grummet J, See AW. Focal low doserate brachytherapy for low to intermediate risk prostate cancer: preliminary experience at an Australian institution. Transl Androl Urol 2021;10(9):3591-3603. doi: 10.21037/tau-21-508 